\title{
New Pathologist Roles In Value-Based Care: Rapid Molecular Pathogen Testing Reduces Hospital Septicemia Costs
}

\section{Eleanor Herriman ${ }^{1}$, Richard Friedberg ${ }^{2}$, Andrew E Horvath ${ }^{3}$, Gary Procop ${ }^{4}$ and Mary Katherine Krause ${ }^{5}$}

${ }^{1}$ Senior Fellow, Harvard Business School, Institute for Strategy and Competitiveness, Ludcke House, 30310 Soldiers Field Road, Boston, MA, USA ${ }^{2}$ Chairman, Department of Pathology Diagnostic Medicine Services Baystate Health Springfield, Massachusetts, Professor and Deputy Chairman, Department of Pathology Tufts University School of Medicine. Massachusetts, USA

${ }^{3}$ Pathology Associates of Albuquerque, PHS-Lab-S1 Albuquerque, NM, USA

${ }^{4}$ Chair, Molecular Pathology, Section Head, Clinical and Molecular Microbiology Director, Parasitology and Mycology Laboratories Professor of Pathology USA

${ }^{5}$ Assistant Professor, Department of Health Systems Management, Rush University; Vice President for CommunicationsCollege of American Pathologists 325 Waukegan Road, Northfield, IL USA

\begin{abstract}
By enabling earlier and more appropriate treatment, rapid molecular pathogen testing (RMPT) can greatly improve the management of acute infectious disorders, such as septicemia. Molecular testing for septicemia offers opportunities to generate substantial value but requires pathologists to expand and/or assume new roles, including:

- Technological and administrative leadership of a complex, rapidly changing field

- Physician education programs to translate science into clinical interventions

- Integration of laboratory and clinical services

- Value-based economic decision-making in a clinical environment.

This paper explores the issues that call for new pathologist roles in molecular pathogen testing, reviews recent literature to assess the value of such testing in treating septicemia, and models potential cost savings for institutions of various sizes.

Two recent U.S. studies of this testing included the impact on hospital costs. Both studies evaluated clinical participation and close collaboration by pharmacists and laboratory professionals in a coordinated septicemia management program. Comparison of patients evaluated with RMPT versus traditional culture-based testing found significant decreases in septicemia-related costs at two hospitals $(30-43 \%$, or approximately $\$ 20,000 /$ patient in direct cost savings). Extrapolating this data to the typical 200-750-bed hospital indicates potential, annual net savings of \$3-6 million per hospital.

Clearly, institutions that implement RMPT for septicemia can generate substantial value, if pathologists expand and/ or assume new roles and responsibilities. Optimally mapping the analytic performance of complex molecular tests to clinical settings with variable types of resistant pathogens and disorders requires the expertise of physician pathologists.
\end{abstract}

Keywords: Septicemia; Meningitis; Microbial pathogens; Genomic testing

\section{Introduction}

Decades of scientific advances have brought modern healthcare to a level of complexity that is having a negative impact on care delivery. "American healthcare is falling short on basic dimensions of quality, outcomes, costs, and equity....The traditional systems for transmitting new knowledge-the ways clinicians are educated, deployed, rewarded, and updated-can no longer keep pace with scientific advances" [1].

Molecular testing highlights this paradox. The technology is advancing exponentially, rapidly creating opportunities for personalized medicine in many therapeutic areas. "Incorporating personalized medicine into the fabric of the healthcare system can help resolve many embedded inefficiencies, such as trial-and-error dosing, hospitalizations due to adverse drug reactions, delayed diagnoses, and reactive treatments. As such, it can also play an important role in the implementation of Accountable Care Organizations set up under the Affordable Care Act to coordinate patient care and reduce costs" [2].

But few providers can keep current with the emerging knowledge base, so opportunities to improve performance are likely being missed. A recent study in a large academic center reported that $40-72 \%$ of all physicians had "no to minimal knowledge" of genomics testing, "with most uncertain when and how to incorporate genomics into practice," and attributed their unpreparedness to lack of knowledge and time to keep up-to-date [3].

Healthcare systems transitioning from fee-for-service to valuebased care payments will need to assess expensive molecular and genomic tests from a different perspective. Despite higher initial costs, some molecular testing applications will generate overall system savings and improve clinical outcomes. A favorable cost-benefit ratio can only be achieved through appropriate test use (i.e. minimization of

${ }^{*}$ Corresponding author: Mary Katherine Krause, Assistant Professor, Department of Health Systems Management, Rush University; Vice President for CommunicationsCollege of American Pathologists 325 Waukegan Road Northfield, IL USA, Tel: 847-832-7461; E-mail: mkkrause@cap.org

Received September 08, 2014; Accepted October 16, 2014; Published October 20, 2014

Citation: Herriman E, Friedberg R, Horvath AE, Procop G, Krause MK (2014) New Pathologist Roles In Value-Based Care: Rapid Molecular Pathogen Testing Reduces Hospital Septicemia Costs. J Health Med Informat 5: 171. doi:10.4172/2157-7420.1000171

Copyright: (c) 2014 Herriman E, et al. This is an open-access article distributed under the terms of the Creative Commons Attribution License, which permits unrestricted use, distribution, and reproduction in any medium, provided the original author and source are credited. 
waste), as well as a timely and effective response to test results. This is why healthcare executives need diagnostic experts to provide scientific and clinical decision-making analytics.

RMPT for sepsis is one such application. A variety of molecular technologies enable patients with septicemia to receive appropriate antibiotics hours or even days sooner, improving outcomes and reducing hospital costs [4] . In these testing programs, pathologists and microbiologists collaborate with infectious disease and pharmacy colleagues to rapidly deliver critical antibiotic information to treating physicians.

"As personalized medicine becomes a reality in mainstream medical practice, physicians and other healthcare providers will have to administer or advise on the application of growing numbers of molecular and genetic tests and pharmacogenomically guided drugs, make treatment decisions based on more predictive evidence and estimations of risk, use information systems for managing patient care, and deal with new ethical and legal issues that arise from molecular and genetic testing." [2].

This paper focuses on one example of the value that healthcare systems can realize from molecular medicine - rapid pathogen identification in sepsis - and describes the increasing clinically and administratively integrated roles that pathologists must assume to capture the opportunity in value-based care settings. Review of recent literature demonstrates the impact in terms of direct hospital cost savings and supports generalization of these findings through economic models that estimate potential savings for healthcare systems.

\section{Background}

Current challenges in sepsis treatment: Sepsis is not only one of the most serious conditions in acute care settings, but also one of the most challenging to treat. "Severe sepsis is common, commonly fatal, difficult to diagnose and easy to mismanage, thus satisfying criteria for an area of clinical practice with a high potential for suboptimal care"[5].

Sepsis ranks among the top 10 most expensive avoidable complications experienced by commercially insured adults, according to analysis by the Prometheus payment system (a claims-based engine for creating bundled episodes of care developed by the Health Care Incentives Improvement Institute and adopted by Medicare). Furthermore, Medicare is incorporating sepsis into its value initiatives, including new outcome measures for Hospital Value Based Purchasing for 2015 [6].

Efforts to improve sepsis care have achieved varying success. The timing and appropriateness of antibiotic treatment are critical. The strongest predictor of survival is the time until initiation of effective antimicrobial therapy; each hour of delay reduces the likelihood of survival by $7.6 \%$ [7]. The current standard of care involves prescribing broad-spectrum antibiotics while awaiting blood culture results, which can take 72 hours or more. This regimen proves ineffective $15-50 \%$ of the time $[7,8]$. "Inadequate or inappropriate treatment is currently seen in $20-25 \%$ of septic patients and is associated with a fivefold reduction in survival"[9].

The traditional blood culture mechanism has other limitations, such as failing to grow organisms in $60-80 \%$ of severely septic patients, contributing to high rates of antibiotic resistant pathogens, and consuming expensive and precious broad-spectrum drugs [9]. For these reasons, new approaches are being sought that identify the pathogen and its susceptibilities more rapidly and accurately.
Rapid pathogen identification in sepsis: Molecular testing to rapidly identify pathogens (RMPT) has recently emerged as a viable clinical tool for guiding antibiotic therapy in acute care settings. "The medical literature has shown that when these rapid tests are used with antimicrobial stewardship programs, there is significant reduction in the time to initiating effective antimicrobial therapy, decreased mortality, and decreased hospital costs"[4].

Current molecular tests analyze pathogens from blood cultures to correctly identify the organism. Some tests may identify multiple organisms or indicate likely antibiotic susceptibility. In general, the benefit of these tests is primarily that of more rapid pathogen identification.

More advanced techniques, such as microarrays, multiplex and quantitative PCR, sequencing and PCR-mass spectrometry ("secondgeneration tests"), analyze pathogens directly from whole blood. They have demonstrated not only cost savings, but also new capabilities and benefits that point to a personalized approach to sepsis management. $[10,11]$ Clinical benefits of these second-generation tests include:

- $\quad$ Monitoring quantitative bacterial load to assess therapeutic response and prognosis [11,12]. Uncovering factors in resistance and virulence [11]

- Identifying patients placed in isolation incorrectly based on false positive results from traditional microbiology tests [11]

- Identifying pathogens in patients whose cultures were negative (false-negatives)

Increasing roles for pathologists: As laboratory-oriented physicians who integrate and interpret complex scientific data and generate clinical reports, pathologists have a central role to play in establishing and operating a successful molecular diagnostics service. Pathologists need to increase their leadership role as pathogen identification transforms from a culture based traditional approach into a complex, value-oriented personalized medicine program.

The value stakes associated with such disorders as sepsis, meningitis, pneumonia, and other disorders are too high not to invest in ensuring implementation of an optimal molecular diagnostics approach. The array of technologies, complexity of their analytic specifications, rapid progression of the field, and capital investment require pathologist leadership and collaboration with clinical and administrative colleagues to ensure that maximum value can be obtained [13-15].

The technical nature of these tests and the likelihood that they will continue as companion diagnostics to traditional blood cultures create the need to have pathologists translate diagnostic information into a clinically meaningful result. This need will only increase as derivative tests emerge, including quantitative pathogen load, resistance patterns, diagnostic next-generation sequencing, and prognostic genetic parameters [16-18].

Pathologists offer value-driven healthcare systems the expert analysis required to select the appropriate molecular and genomic testing platforms, based on the value they generate versus their costs to the laboratory. This unique perspective is essential to aligning incentives properly; otherwise, healthcare systems may not implement programs because their benefits are not visible through a traditional feefor-service, departmental-cost-focused model [19-21]. In addition, it is important to have a pathologist engaged as a member of the healthcare delivery team along with infectious diseases clinicians, pharmacists and 
others to assure that the results from these costly tests are being acted upon in a timely manner to assure maximum impact [22].

\section{Research Methodology}

Our studies included a literature review to identify studies documenting where pathologists have provided RMPT for septicemia and hospital cost impact has been assessed. These studies provided input for a model to estimate potential annual cost savings in hospitals of various sizes.

\section{Study selection}

Studies were identified which had the following criteria;

- A U.S. institution had published the study within the past three years, ensuring accurate reflection of current American healthcare practice.

- At least one of the study authors was a member of a pathology or microbiology department.

- Pathogen identification used a rapid pathogen identification testing method.

- The study assessed the impact of molecular testing on treating septicemia and analyzed direct hospital costs.

The literature review identified two studies that met the search criteria:

- A team at Ohio State University Medical Center conducted a pre- and post-intervention study of patients with Staphylococcus aureus (SA) bacteremia. The study used a rapid polymerase chain reaction (rPCR) test to identify methicillin-resistant $S$. aureus (MRSA) or methicillin-susceptible S. aureus (MSSA) in blood culture bottles that contains gram-positive cocci in clusters [23].

- A team at Methodist Hospital (Houston, Texas) used matrixassisted laser desorption and ionization time-of-flight (MALDITOF) mass spectrometry and rapid antimicrobial susceptibility testing on patients with gram-negative bloodstream infections and conducted a pre- and post-intervention study to measure results [24].

Estimation of potential cost savings for hospitals : The model estimated cost savings for three groups of hospitals, based on bed size:

- Mid-size hospital: 200-400 beds

- Large hospital: more than 400-750 beds

- Major center: more than 750 beds

The model included four inputs for each group of hospitals:

\section{Annual rate of septicemia}

o Healthcare Cost and Utilization Project data (Agency for Healthcare Research and Quality) indicated that $4.2 \%$ of all hospital inpatients received a principal or secondary diagnosis of septicemia [25].

o Discharge data for acute care and children's hospitals from the American Hospital Directory provided the average number of discharges for each hospital group [26].

2. Proportion of septicemia cases suitable for rapid pathogen testing o $75 \%$ of septicemia cases could be candidates for molecular testing based on pathogen type [27].

\section{Average hospital cost for a septicemia stay}

o Data on average cost for hospitals with fewer than 750 beds from the Healthcare Cost and Utilization Project [28].— \$22,000 per case, which was the mean cost per stay for septicemia, updated to 2012 dollars, using the U.S. Consumer Price Index's Inflation Rate for Inpatient Hospital Services [29]. Data on average cost for major centers (above 750 beds) sourced from the Methodist Hospital study. The preintervention mean cost was about $\$ 45,000$.

4. Percentage reduction in hospital costs achievable with rapid pathogen testing services

o Results of the two studies estimated the cost reduction.

\section{Research Results}

In both studies, laboratorians took active, round-the-clock roles in molecular pathogen testing and communicated results rapidly, 24/7, to the infectious disease pharmacist who promptly discussed therapeutic options with the treating physician. The Ohio State University team also communicated results directly and rapidly to the treating physician. The team at Methodist Hospital also conducted regression analysis to identify independent factors associated with decreased length of stay (LOS). This analysis established that the intervention program was an independent factor, apart from multiple co-founders, tied to shorter LOS.

\section{Key study findings}

Both studies found a significant reduction of approximately $\$ 20,000$ in mean hospital costs, representing a 30\% decrease at Ohio State University Medical Center and $43 \%$ at Methodist Hospital (Table 1). Both studies estimated costs on the basis of actual hospital costs for providing care, including all cost centers involved (laboratory, pharmacy, radiology, ICU, etc.).

\section{Potential cost savings for hospitals using rapid pathogen testing for septicemia}

Averaging the results of the two studies indicates that RMPT can reduce hospital costs an estimated $36.5 \%$.

Table 2 shows how the model's inputs are used to estimate cost savings for mid-size hospitals.

Following this methodology, as shown in (Table 3), the estimated cost savings range from $\$ 3$ million to $\$ 6$ million for institutions with 200-750 beds, using an average baseline cost of $\$ 22,000$ for a septicemia stay. The estimated savings increases substantially to $\$ 23$ million for hospitals with more than 750 beds, driven largely by a more-thandouble baseline cost for a septicemia stay [24] and a 65\% increase in the average number of discharges for this hospital group versus large hospitals.

\section{Discussion}

This paper illustrates one example of molecular diagnostics in which pathologists demonstrate a leadership role in increasing value. These two studies identify statistically significant reductions in both time to administration of effective antibiotics and direct hospital costs. The Methodist Hospital study also found that its intervention linked independently with a significant decrease in hospital length of stay, suggesting that these benefits can be attributed to the intervention. Since 


\begin{tabular}{|c|c|c|c|c|c|}
\hline Study & Intervention & $\begin{array}{l}\text { Intervention } \\
\text { cases }\end{array}$ & $\begin{array}{l}\text { Time to effective } \\
\text { antibiotics }\end{array}$ & $\begin{array}{l}\text { Mean length of } \\
\text { stay reduction }\end{array}$ & $\begin{array}{l}\text { Mean hospital cost } \\
\text { reduction per patient }\end{array}$ \\
\hline $\begin{array}{l}\text { Ohio State University } \\
\text { Medical Center [23] }\end{array}$ & $\begin{array}{l}\text { Microbiology lab performs rPCR on positive } \\
\text { cultures and pages results to both infectious } \\
\text { PharmD (during day) and treating physician } \\
\text { ( } 24 / 7 \text { within } 10 \text { minutes) }\end{array}$ & 156 SA bacteremia & $\begin{array}{l}1.7 \text { days, or } 44 \%(p=.002) \\
\text { (MSSA cases) }\end{array}$ & $\begin{array}{l}6.2 \text { days, or } 30 \% \\
(p=.07)\end{array}$ & $\begin{array}{l}\$ 21,387, \text { or } 30 \% \\
(p=.02)\end{array}$ \\
\hline Methodist Hospital [24] & $\begin{array}{l}\text { Microbiology lab performs Mass Spec on positive } \\
\text { cultures and notifies infectious pharmacist } 24 / 7 \\
\text { who advises physician on antibiotics }\end{array}$ & $\begin{array}{l}101 \text { gram-negative } \\
\text { bacteremia }\end{array}$ & $\begin{array}{l}37 \text { hours, or } 51 \%(p<.001) \\
\text { (all intervention patients on } \\
\text { active therapy at } 48 \text { hours) }\end{array}$ & $\begin{array}{l}2.6 \text { days, or } 22 \% \\
(p=.01)\end{array}$ & $\begin{array}{l}\$ 19,547 \text { or, } 43 \% \\
(p=.009)\end{array}$ \\
\hline
\end{tabular}

Table 1: Rapid pathogen identification findings.

\begin{tabular}{|l|c|}
\hline Model input & Value \\
\hline Average number of annual discharges & 12,780 \\
\hline Septicemia cases $=4.2 \%$ of inpatients & 537 \\
\hline$\%$ of septicemia cases suitable for rapid pathogen testing & $75 \%$ \\
\hline Average hospital cost for septicemia & $\$ 22,000$ \\
\hline Cost savings achieved with rapid pathogen testing=37\% per case & $\$ 8,140$ \\
\hline
\end{tabular}

Table 2: Model estimation of cost savings for mid-size hospitals.

\begin{tabular}{|l|c|c|c|}
\hline Hospital group & $\begin{array}{c}\text { Average number } \\
\text { of discharges }\end{array}$ & $\begin{array}{c}\text { Average hospital } \\
\text { cost for septicemia }\end{array}$ & $\begin{array}{c}\text { Potential annual } \\
\text { cost savings }\end{array}$ \\
\hline $\begin{array}{l}\text { Mid-size hospital } \\
\text { (200-400 beds) }\end{array}$ & 12,780 & $\$ 22,000$ & $\sim \$ 3$ million \\
\hline $\begin{array}{l}\text { Large hospital } \\
\text { (>400-750 beds) }\end{array}$ & 23,930 & $\$ 22,000$ & $\sim \$ 6$ million \\
\hline $\begin{array}{l}\text { Major center } \\
(>750 \text { beds) }\end{array}$ & 44,600 & $\$ 45,000$ & $\sim \$ 23$ million \\
\hline
\end{tabular}

Table 3: Estimated cost savings by hospital group.

the completion of their study, the Methodist pathology department has expanded the use of MALDI-TOF testing to all patients with infectious diseases, and is implementing their rapid identification intervention at their affiliated community hospitals [30].

Both studies found trends toward decreased mortality but concluded that sample sizes were too small to establish significance. However, subsequent analysis from Methodist Hospital of their expanded experience with MALDI-TOF rapid testing did show statistically significant decreases in septicemia mortality [30].

Finally, the model's accuracy may be supported by the consistency of its cost savings estimate when using the discharge rate in the American Hospital Directory for Methodist Hospital $(34,280)$ as the model's input. In this case, the model produces an estimated cost savings of $\$ 17,979,000$, which is surprisingly close to the Methodist Hospital team's own comment: "In our 1,000-bed quaternary care hospital, we project a cost savings of $\sim \$ 18$ million annually with the implementation of this strategy for the management of gram-negative BSIs" [24]. The caveat to this comparison is that two of the model's inputs are specific to Methodist Hospital, and thus likely used in their estimate as well.

\section{Other studies}

Previous studies of RMPT for septicemia have also demonstrated both cost savings and survival benefits[31,32]. The test with the most extensive evidence of cost savings is PNA FISH (peptide nucleic acid fluorescent in situ hybridization), one of the earliest molecular methods used in rapid pathogen identification and one of the easiest to implement. A 2010 literature review of septicemia studies using PNA FISH led to the conclusion that "studies evaluating the utility of PNA FISH have shown that it can reduce overall hospital costs by $\$ 4,000$ to $\$ 20,000$, depending on the type of infection. It has also been shown to reduce the amount of antibiotics utilized, as well as decrease mortality"[32].
Another study found a $21 \%$ decrease in median hospital charges using PNA FISH for gram-positive septicemia [33] and yet another reported a $31 \%$ reduction in hospital costs with PNA FISH for Staphylococcus [34], Other studies identified other benefits of rapid molecular testing (using non-FISH tests), including decreased mortality, improved antimicrobial stewardship programs, and lower pathogen resistance rates [4].

Other studies echo the need for pathologists to assume increasing leadership roles in the burgeoning field of microbial identification by molecular diagnostics. As one pathologist stated: "Clinical laboratories must expand their understanding of the complexities related to diagnosing and treating sepsis in order to expand their role as productive members of interdisciplinary healthcare teams focused on improving survival from sepsis and limiting the financial impact that sepsis imparts on our healthcare systems....Laboratory collaboration with emergency medicine departments and critical care services is essential" [31].

This same study emphasized the need for pathologists to take a system-wide perspective on costs. "Clearly, it will be critical to offset costs of new rapid methods with overall reduction of hospital costs. In order for the laboratory to effect these changes, a team approach will rely on interaction with pharmacists, physicians, and other healthcare staff to determine the most judicious use of these methods.... Discussion with healthcare finance and reimbursement teams, as well as antimicrobial utilization teams, are critical for the proper test utilization decisions to be made" [31]

\section{Limitations of This Research}

The decision to limit the literature review to the past three years and to U.S. institutions that analyzed cost savings, so that only two studies met the inclusion criteria, limited the scope of the research. In addition, both studies:

- Used a "pre-versus-post" intervention design, rather than a randomization methodology

- Had relatively small sample sizes (201 and 156 cases)

- Were conducted at large academic institutions, which may limit the ability to reproduce results in other practice settings.

Given these limitations, the institutional cost saving estimates rest on several assumptions that likely have varying validity across institutions. For example, the estimates assume that:

- Septicemia cases are $4.2 \%$ of inpatients

- 75 percent of septicemia cases are targets for rapid pathogen testing

- Average hospital cost for septicemia is $\$ 22,000$ (hospitals 750 beds and under) 
- The net cost reduction achieved with rapid pathogen testing services is $37 \%$

Institutions will have varying rates of septicemia, varying costs for septicemia cases, and varying proportion of their septicemia cases that may be candidates for rapid testing, depending on what testing approach employed. Additionally, depending on the resources available to an institution in terms of personnel and instrumentation investment, the reduction in costs achievable may vary.

Conversely, savings reported in the two studies were net of costs for pathogen testing, as laboratory departmental costs were included in the accounting.

However, the research objective was not to quantify cost savings precisely, but to determine the value represented by molecular testing for septicemia. In that context, the fact that both studies found significant cost savings and that other literature supports their results is a strength of the paper.

\section{Implications and opportunities}

Molecular testing is maturing to the point that valuable clinical applications exist beyond oncology and inherited diseases. RMPT has proven effective at reducing costs and improving outcomes in sepsis, as well as other acute, inpatient disorders with high mortality rates, such as pneumonia and meningitis.

For health administrators pursuing sepsis management programs, the evidence supports at least an evaluation of rapid pathogen identification testing. This innovation offers both primary and secondary benefits, including value generation (net savings with quality improvements) and population health benefits such as lowered resistant rates. Furthermore, as exemplified by Methodist Hospital's decision to apply MALDI-TOF rapid pathogen testing (one of the more capital intensive approaches) across all culture-based infectious testing and at all affiliated community hospitals, there may be an early and substantial return on the required investment for such a program given its many applications and benefits.

Molecular testing for microbial pathogens is not simply a more advanced version of traditional microbiology testing. In development are "genotypic and phenotypic predictors of antibiotic resistance and pharmacogenomic markers of potential drug toxicity" that promise a portfolio of molecular tools for infectious disease treatment [31]. Furthermore, FDA-approved products exist at reasonable costs that afford both molecular microorganism identification, as well as a direct detection of genetic mechanisms of resistance [35-37]. In essence, then, "next generation pathogen testing" represents an entirely new field of personalized medicine that requires complex technical and economic decisions, as well as new laboratory services and team coordination.

As for other areas of personalized medicine, pathologists' implementation of these new services will involve close collaboration with clinicians and administrators. Molecular testing guides treatment decisions, and as such requires that information be delivered in a timely and actionable way. It is unacceptable to have rapid, costly, and actionable molecular diagnostic results lie unattended to in the patient's chart until morning rounds [38]. Particularly with acute infectious disorders, the pathology team must coordinate with others to ensure that treatment recommendations are directly communicated to physicians in a timely, workflow-integrated, patient-centered way. For rapid pathogen testing innovations in septicemia, health care administrators will gain by reaching out for pathologist expertise and leadership to optimize the benefit to healthcare systems [39].

\section{References}

1. Institute of Medicine (2012) Best care at lower cost: The path to continuously learning health care in America.

2. Personalized Medicine Coalition (2011) The Case for Personalized Medicine 3rd edition.

3. Selkirk C, Weissman S, Anderson A, Hulick P (2013) Physicians' Preparedness for Integration of Genomic and Pharmacogenetic Testing into Practice Within a Major Healthcare System. Genet Test Mol Biomarkers 17: 219-225.

4. Goff DA, Jankowski C, Tenover FC (2012) Using rapid diagnostic tests to optimize antimicrobial selection in antimicrobial stewardship programs. Pharmacotherapy 32: 677-687.

5. Huggan PJ (2011) Severe sepsis: take care, take part. Intern Med J 41: 13-8.

6. Center for Medicare and Medicaid Services (2013) National Provider Call: Hospital Value-Based Purchasing. Fiscal Year 2015 Overview for Beneficiaries Providers, and Stakeholders.

7. Kumar A, Roberts D, Wood K, Light B, Parrillo J, et.al (2006) Duration of hypotension before initiation of effective antimicrobial therapy is the critical determinant of survival in human septic shock. Criti Care Med 34: 1589-1596.

8. Lehmann LE, Herpichboehm B, Kost G, Kollef M, Stuber F (2010) Cost and mortality prediction using polymerase chain reaction pathogen detection in sepsis: evidence from three observational trials. Crit Care 14: R186.

9. Lebovitz EE, Burbelo PD (2013) Commercial multiplex technologies for the microbiological diagnosis of sepsis. Mol Diagn Ther 17: 221-231.

10. Alvarez J, Mar J, Varela-Ledo E, Garea M, Matinez-Lamas L, et al. (2012) Cost analysis of real-time polymerase chain reaction microbiological diagnosis in patients with septic shock. Anaesth Intensive Care 40: 958-963.

11. Bhattacharya S (2013) Early diagnosis of resistant pathogens: how can it improve antimicrobial treatment? Virulence 4: 172-184

12. Tran N, Wisner D, Albertson T, Cohen S, Greenhalgh D, et al. (2012) Multiplex Polymerase Chain Reaction Pathogen Detection in Trauma, Emergency, and Burn Surgery Patients with Suspected Septicemia. Surgery 151: 456-463.

13. Callen JL, Westbrook JI, Georgiou A, Li J (2012) Failure to follow-up test results for ambulatory patients: a systematic review. J Gen Intern Med 27: 1334-1348.

14. National Hospital Care Survey (2013) Centers for Disease Control and Prevention.

15. CDC/NCHS National Hospital Discharge Survey (2010) Number and rate of discharges from short-stay hospitals and of days of care, with average length of stay and standard error, by selected first-listed diagnostic categories.

16. Darst JR, Newburger JW, Resch S, Rathod RH, Lock JE (2010) Deciding without data. Congenit Heart Dis 5: 339-342.

17. Forsman $R(2000)$ The electronic medical record: implications for the laboratory. Clin Leadersh Manag Rev 14: 292-295.

18. Kumar A (2011) Optimizing antimicrobial therapy in sepsis and septic shock. Crit Care Nurs Clin North Am 23: 79-97.

19. Laposata M, Dighe A (2007) "Pre-pre" and "post-post" analytical error: highincidence patient safety hazards involving the clinical laboratory. Clin Chem Lab Med 45: 712-719.

20. Levy M, Dellinger R, Townsend S, Linde-Zwirble W, Marshall J, et al. (2010) The Surviving Sepsis Campaign: results of an international guideline-based performance improvement program targeting severe sepsis. Crit Care Med 38 367-374.

21. Loekito E, Bailey J, Bellomo R, Hart G, Hegarty C, et al. (2013) Common laboratory tests predict imminent medical emergency team calls, intensive care unit admission or death in emergency department patients." Emerg Med Australas 25: 132-139.

22. Minton J, Clayton J, Sandoe J, Mc Gann H, Wilcox M (2008) Improving early management of bloodstream infection: a quality improvement project. BMJ 336: $440-443$.

23. Bauer KA, West J, Balada LJM, Pancholi P, Stevenson KB, et al. (2010) An Antimicrobial Stewardship Program's Impact with Rapid Polymerase Chain Reaction Methicillin-Resistant Staphylococcus aureus/S. aureus Blood Culture Test in Patients with S. aureus Bacteremia. Clin Infect Dis 51: 1074-1080. 
Citation: Herriman E, Friedberg R, Horvath AE, Procop G, Krause MK (2014) New Pathologist Roles In Value-Based Care: Rapid Molecular Pathogen Testing Reduces Hospital Septicemia Costs. J Health Med Informat 5: 171. doi:10.4172/2157-7420.1000171

24. Perez KK, Olsen R, Musick W, Cernoch P, Davis J, et al. (2012) Integrating rapid pathogen identification and antimicrobial stewardship significantly decreases hospital costs. Arch Pathol Lab Med 137:1247-1254

25. Elixhauser A, Friedman B, Stranges E (2011) Septicemia in U.S. Hospitals, 2009. Healthcare Cost and Utilization Project.

26. www.ahd.com

27. Morgan M (2013) Clinical Microbiology Newsletter 35:10

28. Pfuntner A, Wier LM, Steiner C (2011) Costs for Hospital Stays in the United States.

29. http://metricmash.com/inflation.aspx?code=SS5702

30. Musser J (2013) Rapid pathogen identification and antimicrobial stewardship associated with decreased hospital costs. Association of Pathology Chairs 2013 Annual Meeting, Boston

31. Wolk DM (2013) Molecular Niches for the Laboratory Diagnosis of Sepsis. Advanced Techniques in Diagnostic Microbiology. 845-871.

32. Eiland E, Beyda N, Han J, Lindgren W, Ward R (2010) The Utility of Rapid Microbiological and Molecular Techniques in Optimizing Antimicrobial Therapy. SRX Pharmacology 2010.

33. Ly T, Gulia J, Pyrgos V, Waga M, Shoham S (2008) Impact upon clinical outcomes of translation of PNA FISH-generated laboratory data from the clinical microbiology bench to bedside in real time. Ther Clin Risk Manag 4 : 637-640.

34. Forrest GN, Mehta S, Weekes E, Lincalis DP, Johnson JK, et al. (2006) Impact of rapid in situ hybridization testing on coagulase-negative staphylococci positive blood cultures. J Antimicrob Chemother 58: 154-158.

35. Wojewoda CM, Sercia L, Navas M, Tuohy M, Wilson D, et al. (2013) Evaluation of the Verigene Gram-positive blood culture nucleic acid test for rapid detection of bacteria and resistance determinants. J Clin Microbiol 51: 2072-2076.

36. Perman SM, Goyal M, Gaieski DF (2012) Initial emergency department diagnosis and management of adult patients with severe sepsis and septic shock. Scand J Trauma Resusc Emerg Med 20: 41.

37. Rivers E, Nguyen B, Havstad S, Ressler J, Muzzin A, et al. (2001) Early goaldirected therapy in the treatment of severe sepsis and septic shock. $\mathrm{N}$ Engl J Med 345: 1368-1377.

38. Roshanov PS, Fernandes N, Wilczynski JM, Hemens BJ, You JJ, et al. (2013) Features of effective computerised clinical decision support systems: metaregression of 162 randomised trials. BMJ 346: f65.

39. Souza J, Beyene H, Van S, Garg A, Haynes R (2013) Features of Effective Computerised Clinical Decision Support Systems: Meta-Regression of 162 Randomised Trials. BMJ 346: f657. 